\title{
The Influence of Previously X-irradiated Aqueous Solutions on the Infectivity of the Viruses of Foot-and-Mouth Disease and Vesicular Stomatitis
}

\author{
By C. D. JOHNSON* \\ Research Institute (Animal Virus Diseases), Pirbright, Surrey
}

(Received 16 April 1964)

SUMMARY

The inactivation of foot-and-mouth disease virus and vesicular stomatitis virus by long-lived inactivating agents in $\mathrm{X}$-irradiated buffer solutions was investigated and compared with the findings of Alper (1952, 1955, 1956) relating to the inactivation of bacteriophage by similar agents. A comparison is made between the effects of these inactivating agents and of hydrogen peroxide to which there is a resemblance. Possible reaction kinetics are discussed in an attempt to explain the form of the survival curves resulting from such inactivation reactions. The radiation sensitization of the viruses to these modes of inactivation is also described and discussed.

\section{INTRODUCTION}

Inactivation of bacteriophage by the long-lived products in $\mathrm{X}$-irradiated aqueous solutions was reported by Alper $(1952,1955,1956)$. She showed that the inactivation of dilute suspensions of bacteriophage in pre-irradiated buffer solutions could not be attributed entirely to the action of formed hydrogen peroxide and that phage, irradiated but still infective, was more susceptible to subsequent inactivation by hydrogen peroxide or by pre-irradiated buffer solution. During investigation of the indirect action of $\mathrm{X}$-irradiation on certain animal viruses a similar behaviour was observed and the results so obtained can be partly explained in terms of reactions at the surface of the virus particle.

\section{METHODS}

Viruses used. Foot-and-mouth disease virus of two immunological types was used: type C strain 997 and type $O$ strain M. 11 (Davie, 1962). Vesicular stomatitis virus of the Indiana type, strain Indiana c, was also used for comparative studies in many experiments.

The foot-and-mouth disease virus was obtained either from the lesions produced following the intradermal inoculation of the plantar pads of guinea pigs or by the propagation of virus on cell monolayers grown from pig kidney (Sellers, 1955) or from a cell line derived from baby hamster kidney (Mowat \& Chapman, 1962; Capstick, Telling, Chapman \& Stewart, 1962). In general the $25 \mathrm{~m} \mu$ infective component of these virus preparations was separated and concentrated by ultracentrifugation (Bradish, Brooksby, Dillon \& Norambuena, 1952) for use in these experiments. This treatment also decreased the concentration of non-viral protein

* Present address: J. J. Thomson Physical Laboratory, University of Reading. 
in the samples. The virus suspensions required for experiment were made up in $0.04 \mathrm{M}$-phosphate buffer $(\mathrm{pH} 7 \cdot 6)$, or $0 \cdot 1 \mathrm{M}$-ammonium bicarbonate adjusted to $\mathrm{pH} 7 \cdot 6$ by acetic acid.

Vesicular stomatitis virus was grown on the chorioallantoic membranes of 7-day developing chick embryos. All data in the text refer to experiments with pooled allantoic and amniotic fluids collected $24 \mathrm{hr}$ after inoculation from batches of about thirty eggs. Because of the sensitivity of vesicular stomatitis virus to light (Skinner $\&$ Bradish, 1954) exposure to light was minimized when handling this virus. The experimental procedure was tested in this respect by using suitable virus controls which were exposed to the same illumination as the virus samples in the main experiment. No significant loss of infectivity was observed in these control samples.

Titration of infectivity. The infectivities of suspensions of foot-and-mouth disease virus were estimated by intraperitoneal inoculation of series of tenfold dilutions into groups of five to ten unweaned mice (Skinner, 1951) or by plaque counts of tenfold dilutions on pig kidney cell monolayers (PK monolayers, Sellers, 1955) or on baby hamster kidney cell monolayers (BHK monolayers, Capstick et al. 1962). A standard inoculum volume of $0 \cdot 3 \mathrm{ml}$. was used in the plaque count technique.

For titration of suspensions of the virus of vesicular stomatitis in mice the intracerebral route of inoculation was used, otherwise the methods of titration were the same as for suspensions of foot-and-mouth disease virus. The results to be described showed no significant dependence on the method of infectivity titration.

In these studies of inactivation the initial infectivity $\left(V_{0}\right)$ and the virus infectivity $V$ surviving a certain inactivation process were the quantities measured. For infectivities measured in mice, $V$ is the reciprocal of the $50 \%$ end-point dilution calculated according to Reed \& Muench (1938). For infectivities measured by plaque assay, $V$ is the number of plaque-forming units counted per $0.3 \mathrm{ml}$. inoculum. The fraction of infectivity surviving, $V / V_{0}$, is used to express the degree of inactivation.

Estimation of hydrogen peroxide. Hydrogen peroxide (British Drug Houses Ltd. Laboratory reagent grade) was used throughout this work. The starch iodide test was used for the detection and determination of hydrogen peroxide at concentrations $10^{-3} \mathrm{M}$ or less (Savage, 1951). A solution of starch + potassium iodide was added to the samples containing hydrogen peroxide and the colour intensity of the blue starch + iodine complex so formed was compared with that of a colour standard produced by adding starch + potassium iodide to known quantities of hydrogen peroxide. This standard was based upon previous titration of hydrogen peroxide against standard ceric sulphate solution. The extinction coefficients were measured on the Coleman Junior Spectrophotometer at $550 \mathrm{~m} \mu$.

$X$-irradiation. All aqueous solutions, including those containing virus, were $\mathbf{X}$ irradiated in closed metal containers lined with borosilicate glass. During irradiation the samples were maintained at temperatures between $0^{\circ}$ and $5^{\circ}$. A Raymax 150 industrial X-ray unit (manufactured by Associated Electrical Industries, London) was used as the source of X-radiation. All irradiations were made with an accelerating voltage of $150 \mathrm{kVp}$ applied to the $\mathrm{X}$-ray tube and with $0.5 \mathrm{~mm}$. duraluminium beam filtration in addition to that provided by the $\mathrm{X}$-ray tube window. This resulted in a beam having a first half-value-layer thickness of $0.5 \mathrm{~mm}$. $\mathrm{Cu}$. 
Irradiation dose rates were 1200 or $3000 \mathrm{r}$./min. according to the specimen container in use. The standard of dose was the ferrous sulphate dosimeter modified by the addition of sodium chloride (Weiss, Allen \& Schwarz, 1955).

Because of the proximity of the specimen containers to the X-ray tube anode there was considerable variation of dose rate with depth within the specimen. Thus under the worst conditions within a specimen depth of $1 \mathrm{~cm}$. at $5 \mathrm{~cm}$. mean distance from the focal spot, the difference in dose rate between the top and bottom of the sample on account of the inverse square law was $40 \%$ of the value at the mean position. Consequently the doses and dose rates quoted in the text refer to mean values derived from measurements of the total dose delivered to a volume of ferrous sulphate irradiated under geometrical conditions identical with those used in the irradiation of virus or other samples.

\section{RESULTS}

Suspensions of foot-and-mouth disease virus and vesicular stomatitis virus were readily inactivated by pre-irradiated buffer solutions provided that the concentration of virus and of non-viral protein or other protective agent was sufficiently low. The same is true of the inactivation of these viruses by hydrogen peroxide. The following features of these reactions were observed.

\section{Inactivation in pre-irradiated buffer}

Pre-irradiated samples of $0.04 \mathrm{~m}$-phosphate buffer, 0.005 M-phosphate buffered saline and $0 \cdot 1 \mathrm{M}$-ammonium bicarbonate all at $\mathrm{pH} 7 \cdot 6$ were capable of inactivating virus to about the same extent. Also, when triple-distilled water was irradiated and then added to $0.4 \mathrm{M}$-phosphate buffer solution so as to produce $0.04 \mathrm{M}$ final concentration at $\mathrm{pH} \mathbf{7 \cdot 6}$, this buffer inactivated virus in a similar manner. Over the reaction times involved in these experiments, normally up to $3 \mathrm{hr}$, the virus suspensions showed no significant decrease of infectivity when mixed with nonirradiated buffer solutions; typical survival curves are shown in Fig. 1. A total of twenty-eight such curves was obtained for various buffer solutions, virus types and irradiation doses, and all were characterized by a slope which decreased continuously with increasing reaction time.

The overall inactivation of a virus suspension for a fixed time in a sample of preirradiated buffer solution was found to depend upon the concentration of virus + residual non-viral protein present in the reaction mixture. This is shown in Fig. 2 in which the extent of inactivation of virus samples of different concentration by identical volumes of irradiated phosphate buffer is related to the concentration of virus used. A similar dependence upon virus concentration was shown when the inactivating agent was hydrogen peroxide.

\section{Protection effects}

Protein or other protective agents such as glutathione or thiourea, in the absence of any toxic effects, decreased the rate of inactivation of virus infectivity in preirradiated buffer solution. When present in sufficiently high concentrations, these agents annulled any observable inactivation. This effect is shown in Table 1 which relates to the inactivation of vesicular stomatitis virus by pre-irradiated phosphate 
buffered saline. The infectivity remaining after reaction for $2 \mathrm{hr}$ at $4^{\circ}$ in the presence of different concentrations of peptone increased with the concentration of peptone. At peptone concentrations in excess of $1 \mathrm{~g} . / \mathrm{l}$. no significant inactivation was detected. Thus a convenient method of studying reaction rates is to take samples from the reaction mixture at appropriate times and to stop the reaction in these

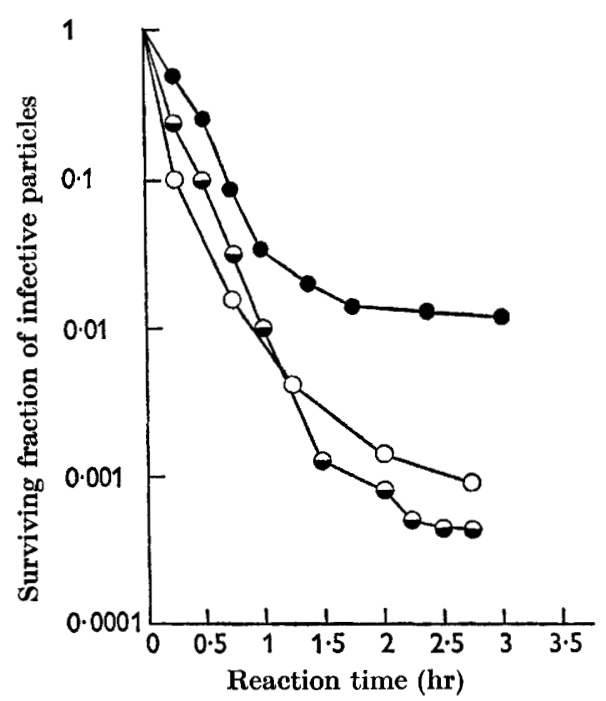

Fig. 1

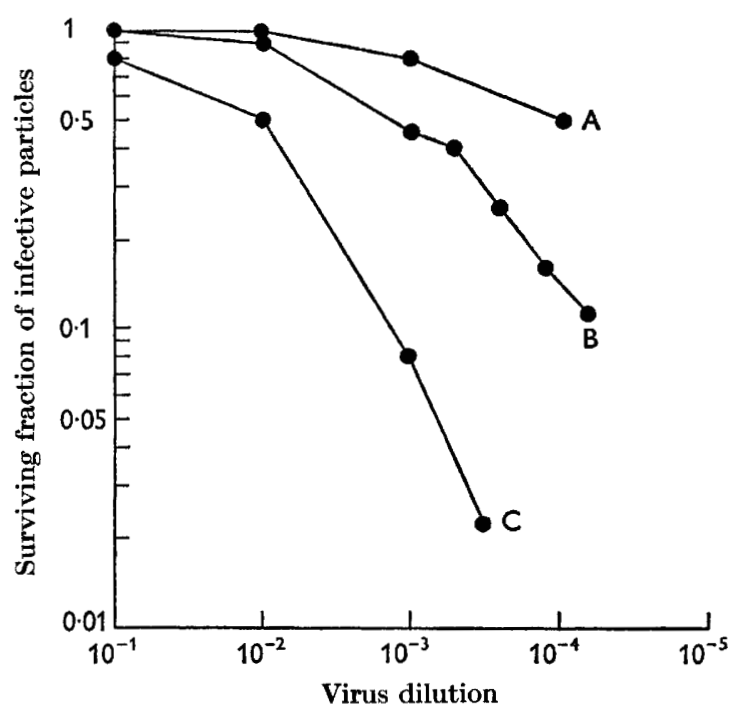

Fig. 2

Fig. 1. Typical survival curves for the inactivation of foot-and-mouth disease (FMD) and vesicular stomatitis (VS) viruses by $\mathrm{X}$-irradiated buffer solutions. Irradiation dose $=30,000 \mathrm{r}$. O, Inactivation of FMD virus by irradiated $0 \cdot 1 \mathrm{~m}$-ammonium bicarbonate buffer. $O$, Inactivation of FMD virus by irradiated $0.005 \mathrm{M}$-phosphate buffered saline. $\odot$, Inactivation of VS virus by irradiated $0.005 \mathrm{M}$-phosphate buffered saline. All titrations of infectivity on pig kidney cell monolayers.

Fig. 2. Inactivation of various concentrations of foot-and-mouth disease virus in hydrogen peroxide and pre-irradiated buffer solutions. Constant reaction time of $3 \mathrm{hr}$ at $4^{\circ}$. Infectivities titrated on pig kidney cell monolayers. A, Inactivation in $10^{-3.5} \mathrm{M}$-hydrogen peroxide; $B$, inactivation in $0.04 \mathrm{M}$-phosphate buffer irradiated for $30 \mathrm{~min}$. at $3000 \mathrm{r} . / \mathrm{min}$.; $\mathrm{C}$, inactivation in $10^{-3 \cdot 0} \mathrm{M}-\mathrm{hydrogen}$ peroxide.

Table 1. Depression of infectivity of vesicular stomatitis virus in egg fluids titrated on pig kidney cell monolayers following reaction for $3 \mathrm{hr}$ with pre-irradiated phosphate buffered saline in the presence of various concentrations of peptone at $p H \mathbf{H} 6$

$\begin{array}{cr}\begin{array}{c}\text { Concentration of } \\ \text { peptone in buffer }\end{array} & \begin{array}{r}\text { Surviving fra } \\ \text { of infecti }\end{array} \\ \text { g./. } & V / V_{0} \\ 100 & 0 \cdot 95 \\ 30 & 1 \cdot 10 \\ 10 & 1 \cdot 00 \\ 3 & 1 \cdot 05 \\ 1 & 0 \cdot 95 \\ 0.1 & 0 \cdot 8 \\ 0.01 & 0.55 \\ \text { Nil } & 0.10\end{array}$


samples by the addition of an equal volume of, say, a peptone solution at a concentration of $10 \mathrm{~g} . / \mathrm{l}$. (pH 7.6). The whole set of samples may then be titrated for infectivity at the termination of the reaction.

\section{Rates of inactivation}

Apart from the dependence upon concentration of reactants the rate of inactivation of the viruses in pre-irradiated buffer solutions was found to increase with the temperature of the reaction mixture and to decrease with the quantity of air or

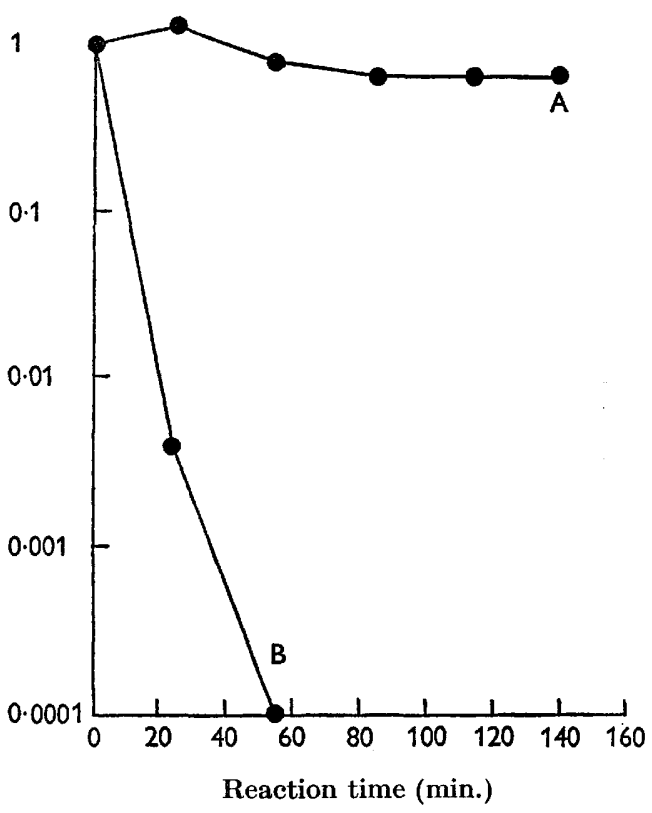

Fig. 3

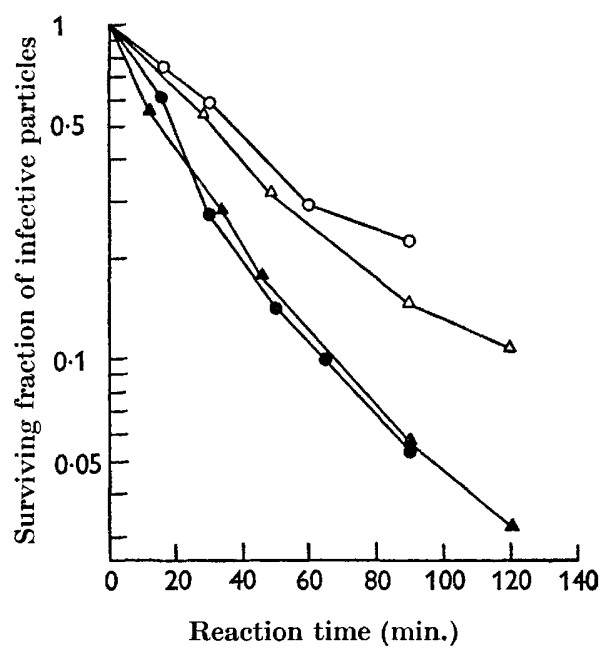

Fig. 4

Fig. 3. Inactivation of vesicular stomatitis virus by pre-irradiated phosphate buffer solutions. A, buffer X-irradiated in frozen state at $-60^{\circ}$ for $30 \mathrm{~min}$. at $3000 \mathrm{r} . / \mathrm{min}$.; $B$, buffer solution irradiated in liquid state for $30 \mathrm{~min}$. at $3000 \mathrm{r} . / \mathrm{min}$.

Fig. 4. Inactivation of vesicular stomatitis (VS) virus in irradiated phosphate buffered saline $(O)$ and in a solution of hydrogen peroxide $(\triangle)$ compared with the inactivation of the pre-irradiated material by the same two inactivating agents, points $\bullet$ and $\Delta$. The concentration of hydrogen peroxide was arbitrarily selected to give approximately the same rate of inactivation as the irradiated buffer solution. Irradiation doses to buffer solution and virus were each $3 \times 10^{4} \mathrm{r}$. $\triangle$, Inactivation of VS virus by $10^{-3} \mathrm{M}$-hydrogen peroxide; $O$, inactivation of VS virus by irradiated phosphate buffered saline; $\Delta$, inactivation of irradiated VS virus by $10^{-3} \mathrm{M}$-hydrogen peroxide; $\boldsymbol{O}$, inactivation of irradiated VS virus by irradiated phosphate buffered saline.

oxygen dissolved in the buffer solution at the time of irradiation. All comparisons between rates of reaction subsequently given are for reactions at $4^{\circ}$ with airsaturated buffer solutions. No inactivation was observed when buffer solution irradiated in the frozen state was thawed and used with virus at $4^{\circ}$. This is shown in Fig. 3; the upper curve shows no significant loss of infectivity for the reaction between vesicular stomatitis virus and phosphate buffer pre-irradiated at about $-60^{\circ}$; the lower curve shows the same virus treated with buffer solution preirradiated at a temperature between $0^{\circ}$ and $5^{\circ}$. 


\section{Virus sensitization effect}

The $\mathbf{X}$-irradiation of virus suspensions rendered them more susceptible to subsequent inactivation by pre-irradiated buffer solution and to the action of hydrogen peroxide. Also pre-treatment of virus suspensions with hydrogen peroxide rendered them more sensitive to inactivation by pre-irradiated buffer. These effects have been termed virus sensitization (Alper, 1952). The effect of increasing the susceptibility of virus to the inactivating agents of pre-irradiated buffer and to hydrogen peroxide was shown in a set of experiments of which the results are shown in Figs. 4-6.

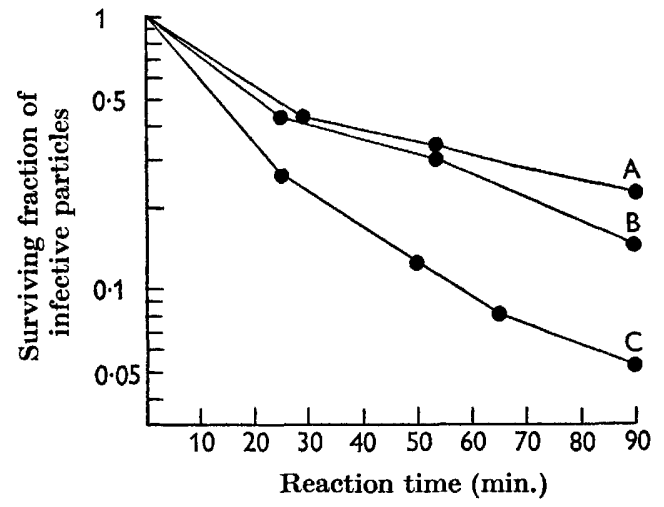

Fig. 5

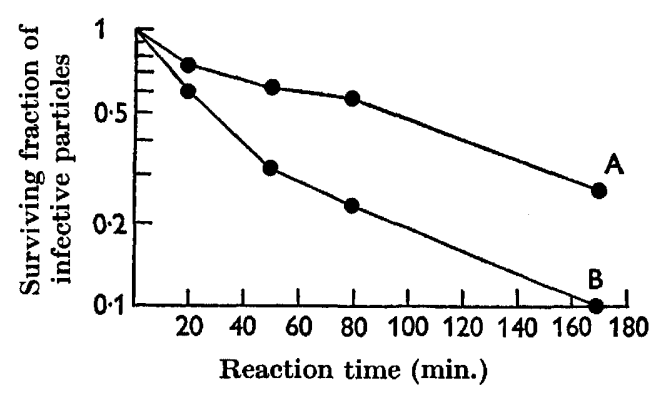

Fig. 6

Fig. 5. Inactivation of irradiated vesicular stomatitis (VS) virus in pre-irradiated phosphate buffered saline. Infectivities titrated on baby hamster kidney cell monolayers. $A$, inactivation of non-irradiated VS virus; $B$, inactivation of VS virus pre-irradiated for $30 \mathrm{~min}$. at $3000 \mathrm{r}$./min.; C, inactivation of VS virus pre-irradiated for $90 \mathrm{~min}$. at $3000 \mathrm{r}$./ min.

Fig. 6. Inactivation of stomatitis (VS) virus in pre-irradiated phosphate buffered saline before and after treatment with hydrogen peroxide. Infectivities titrated on baby hamster kidney monolayers. A, VS virus inactivated by pre-irradiated phosphate buffered saline; $B$, VS virus treated with hydrogen peroxide and subsequently inactivated by pre-irradiated phosphate buffered saline.

A dose of $3 \times 10^{4}$ r. of $X$-radiation was delivered to a $1 / 10$ dilution of pooled egg fluids infected with vesicular stomatitis virus. This resulted in a $60 \%$ loss of infectivity, probably due primarily to the action of short-lived free radicals in solution (Lea, 1946). The irradiated virus was then immediately further diluted 1/300 into: (a) phosphate buffer which had received an irradiation dose of $3 \times 10^{4} \mathrm{r}$. at the same time as the virus itself; $(b)$ into hydrogen peroxide diluted to $10^{-3} \mathrm{M}$ in phosphate buffer solution. Similar reaction mixtures were prepared from non-irradiated virus. Fig. 4 shows the resulting inactivation of virus in the four reaction mixtures. It is apparent that $10^{-3} \mathrm{M}$-hydrogen peroxide inactivated the virus at a very similar rate to that of the pre-irradiated buffer and that in each case the pre-irradiation of the virus suspensions resulted in an increased rate of inactivation. The concentration of hydrogen peroxide in the four reaction mixtures was determined at the start and end of the reaction time by the starch iodide test. The concentration of hydrogen peroxide, as detected by this test, was more than one hundred times greater in the samples containing $10^{-3} \mathrm{M}$-hydrogen peroxide than in those containing pre-irradi- 
ated buffer solution. Also the concentration of hydrogen peroxide did not decrease significantly during the reaction, but in the case of pre-irradiated buffer the concentration of formed hydrogen peroxide was near the limit of detection. Thus this observation could not be made fully quantitative. This experiment was repeated with only irradiated phosphate buffer as the inactivating agent, but two identical samples of virus were pre-irradiated for different times at the same dose rate. The subsequent rates of inactivation of the surviving virus were compared with that of the non-irradiated virus in the control samples. The rate of inactivation increased with the extent of pre-irradiation (Fig. 5).

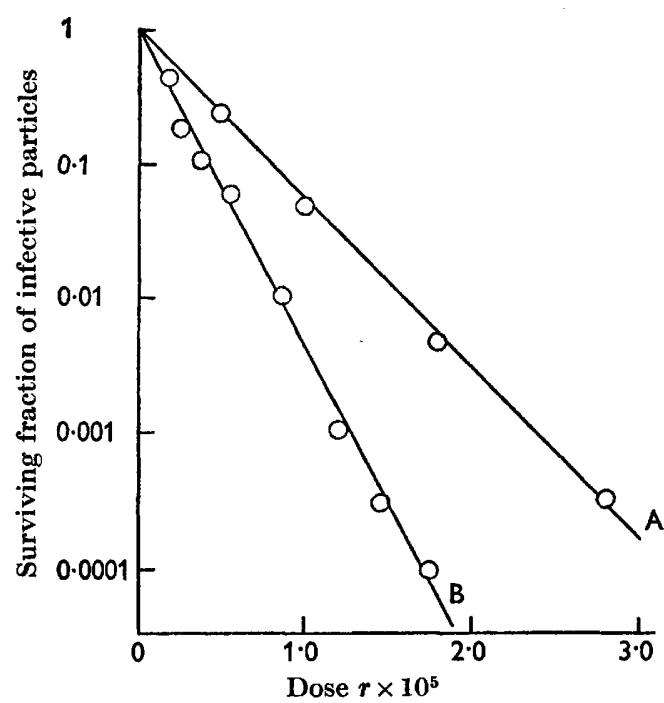

Fig. 7

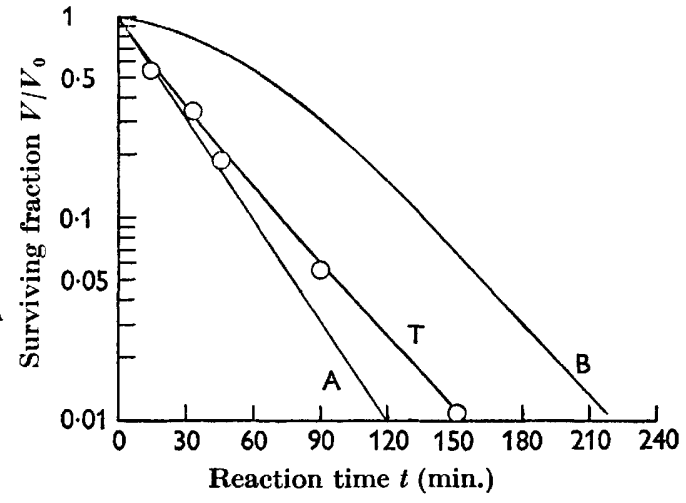

Fig. 8

Fig. 7. Inactivation by the indirect effect. A, inactivation of foot-and-mouth disease (FMD) virus (strain c/997) by $\mathrm{X}$-irradiation in aqueous solution of low non-viral protein concentration; infectivities titrated in unweaned mice. $B$, inactivation of vesicular stomatitis (VS) virus (Indiana) by $\mathbf{X}$-irradiation in aqueous solution of low non-viral protein concentration; infectivities titrated on baby hamster kidney cell monolayers.

Fig. 8. Theoretical survival curve $T$ derived by combining curve $A$ for a single-step mechanism with curve $B$ for a three-step mechanism, weighted in the ratio 9:1. The experimental points are for the inactivation of vesicular stomatitis (VS) virus by irradiated phosphate buffered saline. Curve $A$ has the equation $V / V_{0}=e^{-k t}$ and curve $B$ has the equation $V / V_{0}=e^{-k t}\left(1+k t+\frac{1}{2} k^{2} t^{2}\right)$ where $k$ has the numerical value 0.033 when time $t$ is in minutes.

To examine whether virus sensitization could be produced by pre-treatment of virus with hydrogen peroxide, a suspension of vesicular stomatitis virus was diluted $1 / 10$ in $10^{-3} \mathrm{M}$-hydrogen peroxide. After $3 \mathrm{hr}$ at $4^{\circ}$ this virus suspension showed a $20 \%$ loss of infectivity, indicating that sufficient hydrogen peroxide had been used to produce a significant effect. However, when further diluted 1/300 in irradiated phosphate buffer solution, this treated virus was inactivated much more rapidly than normal virus at the same overall dilution. The results of an experiment of this type are shown in Fig. 6.

It may be noted that the high dose rates used in these irradiations were necessary 
in order that the time of irradiation should be short compared with the time required for observation of the inactivation by the long-lived agents. In this way the two distinct modes of inactivation, the instantaneous indirect effect and the postirradiation effect, were separated. In the discussion of the effects of pre-irradiation on virus samples it is justifiable to assume that the depression of infectivity caused by the irradiation was almost wholly due to the indirect effect of short-lived free radicals in solution.

As a supplement to the data on post-irradiation effects, typical exponential survival curves for the inactivation of the infectivity of suspensions of foot-andmouth disease virus and vesicular stomatitis virus by free radicals in aqueous solutions are presented in Fig. 7. These curves were obtained by irradiating aqueous suspensions of the viruses at high dose rates. Samples of virus taken for titration of infectivity during the irradiation were immediately diluted into a solution of peptone $(50 \mathrm{~g} . / 1$.) in order to prevent any subsequent inactivation by the postirradiation effects. Exponential survival curves result from this mode of inactivation.

\section{DISCUSSION}

From the data presented here three main points emerge. First, in connexion with the effect of hydrogen peroxide solutions on virus infectivity, it is clear that there are some similarities between hydrogen peroxide and the inactivating agents present in X-irradiated buffer solutions. One agent responsible for inactivation in the latter case may be formed hydrogen peroxide. There is, however, a considerable quantitative difference between the amount of hydrogen peroxide formed in an $\mathrm{X}$-irradiated buffer solution and that which must be added to a non-irradiated buffer solution in order to achieve the same rate of inactivation. This difference has been attributed to the presence of stabilizers in commercial preparations of hydrogen peroxide (Alper, 1954).

The second observation, which is of more immediate interest in virology, is that $\mathrm{X}$-irradiation in aqueous solution can modify the viruses of foot-and-mouth disease and vesicular stomatitis in such a way that, although still infective, they become more sensitive to the subsequent action of hydrogen peroxide or irradiated buffer solution. This implies a modification of the intact virus particle of such a nature that its ability to infect the animal cell is not impaired. As this modification is probably brought about by free radicals in solution and as some free radicals can also themselves produce inactivation, it is reasonable to suppose that the virus sensitization effect is a result of those reactions between the virus and the free radicals which do not result in direct inactivation. It is thought that there are many such reactions for every one which causes an inactivation (Allen, 1961).

The third observation relates to the shape of the inactivation curves which, as shown in Fig. 1, are non-linear and in all cases show a gradual decrease in rate of inactivation as the reaction proceeds. When inactivation of virus is caused by the indirect effect, exponential survival curves are obtained for both foot-and-mouth disease virus and vesicular stomatitis virus (Fig. 7). The reaction kinetics which describe this form of inactivation make the following assumptions (Allen, 1961): (1) That a constant dose rate is maintained and hence a constant free radical concentration exists during the inactivation. (2) That the virus particle is capable of 
reacting with free radicals to produce either loss of infectivity or other modifications which may not be detected. (3) That inactivation results from reactions of the single-event type occurring at random among the other reactions. (4) That a virus after inactivation continues to react as readily with the free radicals as it did before the inactivation. If the post-irradiation inactivation process were due to a similar single-event interaction produced by a constant concentration of inactivating agents capable of combining with infective and non-infective virus alike, then exponential survival curves would be expected. The observation that such curves are not obtained implies that one or more of the conditions set out above is not fulfilled.

The shape of the survival curves is generally consistent with what might be expected if the concentration of inactivating agents became less, either as a result of loss due to recombination or decay, or as a result of combination with virus and other protein within the reaction mixture. It was shown, however, that inactivation curves of the same characteristic shape were obtained when $10^{-3} \mathrm{M}$-hydrogen peroxide was used as the inactivating agent (Fig. 4) and also that in this instance the concentration of hydrogen peroxide remained unchanged during the course of the reaction. Nevertheless, it is possible that, when the inactivation is due to agents in $\mathrm{X}$-irradiated buffer solution, the inactivating agent does, in fact, become less concentrated during the reaction; and in the experiment with hydrogen peroxide mentioned above, some other agent, undetected by the starch iodide test, might account for the inactivation. It might be possible for this associated agent to become less concentrated during the reaction, thus producing a survival curve of the observed shape, without any apparent decrease in the concentration of hydrogen peroxide itself. This might also partly account for the relatively high concentration of hydrogen peroxide needed to simulate the effect of irradiated buffer solutions.

An alternative explanation of the shape of the survival curves is that the inactivation is in part a multi-step process. Fig. 8 shows how a virus sample of which $90 \%$ is inactivated by a single-step reaction and $10 \%$ by a cumulative three-step reaction can result in an overall survival curve of the observed shape. There are many parameters in an analysis of this type; for example, the reaction constant $k$ may not be the same for the one-step and the three-step reactions, and higher-order reactions may be involved. Consequently a considerable variety of theoretical shapes is possible when the different processes are combined. On a model of this form it is necessary to assume that the proportion of virus particles which are inactivated by, say, the three-step process cannot be inactivated by the single-step mechanism, otherwise the latter would dominate. In other words there are different degrees of sensitivity to inactivation by irradiated buffer within the virus sample. The one-step reaction may be merely the last stage of the multi-step reactions. Another possibility is that the inactivating agents in irradiated buffer do not combine equally readily with infective and non-infective virus, thus modifying the reaction kinetics as the ratio of infective to non-infective particles changes.

Any acceptable model of the types outlined above must attempt to explain the virus sensitization effect which is observed in parallel with the inactivation process. Although it is difficult to correlate the virus sensitization effect with a single-step inactivation mechanism, the effect can be broadly accounted for in terms of the multi-step inactivation mechanism. If the mean number of steps required to 
produce inactivation in a given sample is decreased then there will be an overall increase in the rate of inactivation compared with that of the original virus sample. There may also be some change in the shape of the inactivation curve, but consideration of this is outside the accuracy and scope of this work.

If, as a result of sensitization irradiation, a virus suspension had lost $60 \%$ of its initial infectivity by the indirect effect, the residual infective virus would have combined with free radicals in the types of reaction not producing direct inactivation. These reactions might have modified the virus sample in such a way that the relative population of infective particles requiring only single-step inactivation in pre-irradiated buffer was increased. This would have the net effect of increasing the sensitivity of the virus sample to inactivation by pre-irradiated buffer. It would therefore be expected that the degree of sensitization would depend upon the preirradiation dose delivered to the virus; this was shown to be so in Fig. 6. There is an obvious limit to the amount of pre-irradiation which may be employed without destroying the majority of the virus infectivity.

It is interesting to speculate on the possibilities that the virus sensitization effect may be demonstrated by means other than these observations on the rate of inactivation in hydrogen peroxide or pre-irradiated buffer solutions. It is conceivable that the specific reactions between virus and its antiserum may be altered by irradiation treatment of the virus. This treatment may also modify the sensitivity to thermal and other degradation processes which influence the complex surface of the virus particle.

I wish to thank Miss H. Wigman, Miss R. Jowett and Mr M. Ryal for their valuable assistance; Miss H. E. N. Ferrier for supplying much of the virus used, and Dr C. J. Bradish for his advice and encouragement.

\section{REFERENCES}

Allen, A. O. (1961). Radiation Chemistry of Water and Aqueous Solutions. Toronto: D. Van Nostrand Co.

Alper, Tikvah (1952). A new after-effect of X-rays on dilute aqueous suspensions of bacteriophage. Nature, Lond. 169, 964.

Alper, Tikvar (1954). The inactivation of free bacteriophage by irradiation and by chemical agents. J. gen. Microbiol. 11, 313.

Alper, Tikvar (1955). Bacteriophage as an indicator in radiation chemistry. Rad. Res. $2,119$.

Alper, Tikvah (1956). The modification of damage caused by primary ionization of biological targets. Rad. Res. 5, 573.

Bradish, C. J., Brooksby, J. B., Dillon, J. F. \& Norambuena, M. (1952). Ultracentrifugation studies of the infective and complement-fixing components in the virus system of foot-and-mouth disease. Proc. Roy. Soc. B, 140, 107.

Capstick, P. B., Teluing, R. C., Chapman, W. G. \& Stewart, Doreen L. (1962). Growth of a cloned strain of hamster kidney cells in suspended cultures and their susceptibility to the virus of foot-and-mouth disease. Nature, Lond. 195, 1163.

Davie, J. (1962). The classification of subtype variants of the virus of foot-and-mouth disease. Report Off. int. Epizoot. No. 169.

LEA, D. E. (1946). Actions of Radiations on Living Cells, 1st edn. Cambridge University Prèss.

Mowat, G. N. \& Chapman, W. G. (1962). Growth of foot-and-mouth disease virus in a fibroblastic cell line derived from hamster kidneys. Nature, Lond. 194, 253. 
Reed, L. T. \& Muench, H. (1938). A simple method of estimating 50 per cent end-points. Amer. J. Hyg. 27, 493.

SAvAGE, D. J. (1951). The determination of hydrogen peroxide in radiation experiments on aqueous solutions. Analyst, 76, 224.

SELLERS, R. F. (1955). Growth and titration of the virus of foot-and-mouth disease and vesicular stomatitis in kidney monolayer tissue cultures. Nature, Lond. 176, 547.

Skinner, H. H. (1951). Propagation of strains of foot-and-mouth disease in unweaned mice. Proc. Roy. Soc. Med. 44, 1041.

Skinner, H. H. \& Bradish, C. J. (1954). Exposure to light as a source of error in the estimation of the infectivity of virus suspensions. J. gen. Microbiol. 10, 377.

Weiss, J., Allen, A. O. \& Schwarz, H. A. (1955). Use of the Frick ferrous sulphate dosimeter for gamma-ray doses in the range 4 to $40 \mathrm{kr}$. Int. Conf. Peaceful Uses of Atomic Energy, 14, 179. 
